\title{
A $K$-FUNCTIONAL AND THE RATE OF CONVERGENCE OF SOME LINEAR POLYNOMIAL OPERATORS
}

\section{Z. DITZIAN}

(Communicated by J. Marshall Ash)

Abstract. We show that the $K$-functional

$$
K\left(f, n^{-2}\right)_{p}=\inf _{g \in C^{2}[-1,1]}\left(\|f-g\|_{p}+n^{-2}\|P(D) g\|_{p}\right),
$$

where $P(D)=\frac{d}{d x}\left(1-x^{2}\right) \frac{d}{d x}$, is equivalent to the rate of convergence of a certain linear polynomial operator. This operator stems from a Riesz-type summability process of expansion by Legendre polynomials. We use the operator above to obtain a linear polynomial approximation operator with a rate comparable to that of the best polynomial approximation.

\section{INTRODUCTION}

For polynomial approximation in $L_{p}[-1,1], 1 \leq p \leq \infty$, it was shown [6, Chapter 7] that

$$
E_{n}(f)_{p} \equiv \inf _{P \in \Pi_{n}}\|f-P\|_{p} \leq \inf _{g \in C^{r}[-1,1]}\left(\|f-g\|_{p}+n^{-r}\left\|\varphi^{r} g^{(r)}\right\|_{p}\right) \equiv K_{r, \varphi}\left(f, n^{-r}\right)_{p}
$$

where $\varphi=\sqrt{1-x^{2}}, \Pi_{n}$ is the class of polynomials of degree $n$ and \|\|$_{p}$ is the $L_{p}[-1,1]$ norm. In fact, a polynomial $P \in \Pi_{n}$ was constructed to satisfy

$$
\|f-P\|_{p} \leq K_{r, \varphi}\left(f, n^{-r}\right)_{p} .
$$

The result (1.1) is best possible in the sense that a weak converse inequality for $E_{n}(f)_{p}$ exists. As a result of the considerations in this paper we obtain

$$
E_{n}(f)_{p} \leq \inf _{g \in C^{2 r}[-1,1]}\left(\|f-g\|_{p}+n^{-2 r}\left\|P(D)^{r} g\right\|_{p}\right) \equiv K_{2 r}\left(f, n^{-2 r}\right)_{p}
$$

(where $P(D)=\frac{d}{d x}\left(1-x^{2}\right) \frac{d}{d x}$ ), and this inequality is best possible in the same sense, that is, a weak converse inequality with $E_{n}(f)_{p}$ is valid. The $K$-functionals $K_{2 r, \varphi}\left(f, n^{-2 r}\right)_{p}$ and $K_{2 r}\left(f, n^{-2 r}\right)_{p}$ are not equivalent though related for $1<p<\infty$ (see for explicit results $[3,(2.5)]$ in the case $r=1$ ). For $r=1$ and $f(x)=x$,

Received by the editors April 6, 1994 and, in revised form, November 18, 1994.

1991 Mathematics Subject Classification. Primary 41A10, 41A35, 41A25.

Key words and phrases. Linear polynomial approximation, near best polynomial approximation.

Supported by NSERC grant A4816 of Canada.

(C)1996 American Mathematical Society 
$K_{2, \varphi}\left(f, n^{-2}\right)_{p}=0$ and $K_{2}\left(f, n^{-2}\right)_{p} \sim n^{-2}$. Hence, (1.2) cannot be derived from (1.1) or vice versa.

For Bernstein polynomials

$$
B_{n}(f, x)=\sum_{k=0}^{n}\left(\begin{array}{l}
n \\
k
\end{array}\right) x^{k}(1-x)^{n-k} f\left(\frac{k}{n}\right) \equiv \sum_{k=0}^{n} P_{n, k}(x) f\left(\frac{k}{n}\right),
$$

it was shown by Totik [9] (generalizing [7, section 8] and settling a conjecture there) that

$$
\left\|f-B_{n} f\right\|_{C[0,1]} \sim K_{2, \varphi}\left(f, n^{-1}\right)_{\infty}
$$

where $L_{\infty}[0,1]$ takes the place of $L_{\infty}[-1,1]$ and $\varphi=\sqrt{x(1-x)}$ takes the place of $\varphi=\sqrt{1-x^{2}}$ in the definition of the $K$-functional in (1.1). Hence,

$$
E_{n}(f)_{C[0,1]} \leq C\left\|f-B_{n^{2}} f\right\|_{C[0,1]} .
$$

For $p \neq \infty$ and for a higher degree of smoothness, the Bernstein polynomials are no longer applicable. In this case one can define the Durrmeyer-Bernstein polynomials

$$
M_{n}(f, x)=\frac{1}{n+1} \sum_{k=0}^{n} P_{n, k}(x) \int_{0}^{1} P_{n, k}(y) f(y) d y .
$$

As a result of [4] we have for $1 \leq p \leq \infty$

$$
K_{2 r}\left(f, n^{-r}\right)_{p} \sim\left\|\left(M_{n}-I\right)^{r} f\right\|_{p}
$$

where in the definition of $K_{2 r}$ we use $L_{p}[0,1]$ instead of $L_{p}[-1,1]$ and $P(D)=$ $\frac{d}{d x} x(1-x) \frac{d}{d x}$ instead of $P(D)=\frac{d}{d x}\left(1-x^{2}\right) \frac{d}{d x}$. The result of this paper will imply for $1 \leq p \leq \infty$ and $r \in N$

$$
E_{n}(f)_{L_{p}[0,1]} \leq C\left\|\left(M_{n^{2}}-I\right)^{r} f\right\|_{L_{p}[0,1]} .
$$

To achieve this we define a simple linear operator $R_{n}$ that will yield the strong converse inequality of type $\mathrm{A}$ in the sense of [7], and hence the equivalence

$$
\left\|\left(R_{n}-I\right)^{r} f\right\|_{L_{p}[-1,1]} \sim K_{2 r}\left(f, n^{-2 r}\right)_{p} .
$$
by

$$
P(D) P_{k}(x) \equiv \frac{d}{d x}\left(1-x^{2}\right) \frac{d}{d x} P_{k}(x)=-k(k+1) P_{k}(x)
$$

and the orthonormality condition

$$
\left\langle P_{k}, P_{\ell}\right\rangle \equiv \int_{-1}^{1} P_{k}(x) P_{\ell}(x) d x=\delta_{k, \ell}
$$

appear naturally. Therefore, the operator $P(D)$ is not a contrived expression in the definition of $K_{2 r}$ and in (1.2).

In section 2 the operator $R_{n} f$ will be introduced. In section 3 the main converse inequality is proved. In section 4 applications and generalizations of this result will be discussed. The relation with best polynomial approximation is given in section 5 . 


\section{A summability operator}

For the Legendre polynomial $P_{k}$ given in (1.10) and (1.11) the formal expansion is given by

$$
f(x) \sim \sum_{k=0}^{\infty} a_{k} P_{k}(x), \quad a_{k}=\left\langle f, P_{k}\right\rangle=\int_{-1}^{1} f(y) P_{k}(y) d y .
$$

Pollard [8] showed that for $\frac{4}{3}<p<4$

$$
\left\|S_{n} f\right\|_{p} \leq C_{p}\|f\|_{p}
$$

where

$$
S_{n}(f, x)=\sum_{k=0}^{n} a_{k} P_{k}(x)
$$

Askey and Hirschman [2] (see Theorem 2a there with $\alpha=1$ and $\nu=\frac{1}{2}$ ) proved that for $1 \leq p \leq \infty$

$$
\left\|\sigma_{n} f\right\|_{p} \leq C\|f\|_{p}
$$

where $\sigma_{n} f$ is the Cesàro summability

$$
\sigma_{n-1}(f, x)=\sum_{k=0}^{n}\left(1-\frac{k}{n}\right) a_{k} P_{k}(x)=\frac{1}{n} \sum_{k=0}^{n-1} S_{k}(f, x), \quad \sigma_{0}(f, x)=a_{0} P_{0} .
$$

The summability method treated here is given by

$$
R_{n}(f, x)=\sum_{k=0}^{n}\left(1-\frac{k(k+1)}{n(n+1)}\right) a_{k} P_{k}(x)
$$

and (2.6) may be reformulated by

$$
R_{n}(f, x)=\frac{2}{n(n+1)} \sum_{k=0}^{n-1}(k+1) S_{k}(f, x),
$$

which places it as a special case of the Riesz summability. Being a linear operator whose range is in $\Pi_{n}, R_{n} f$ and its derivates are linear polynomial operators.

We can now show that, as a result of (2.4), $R_{n} f$ is also a bounded operator.

Lemma 2.1. For $1 \leq p \leq \infty$ and $R_{n}$ given by (2.6) we have

$$
\left\|R_{n} f\right\|_{p} \leq C\|f\|_{p}
$$

Proof. The following proof was given by V. Totik and is much shorter than the original proof by the author. (The earlier proof was longer and yielded some further results which are not necessary here.) 
One can easily verify that

$$
\frac{2}{n(n+1)} \sum_{k=0}^{n-1}(n-k) S_{k}(f, x)=\frac{2}{n(n+1)} \sum_{k=0}^{n-1}(k+1) \sigma_{k}(f, x) .
$$

Adding (2.9) to (2.7), we have

$$
\frac{2}{n(n+1)}(n+1) \sum_{k=0}^{n-1} S_{k}(f, x)=R_{n}(f, x)+\frac{2}{n(n+1)} \sum_{k=0}^{n-1}(k+1) \sigma_{k}(f, x),
$$

which implies

$$
R_{n}(f, x)=2 \sigma_{n-1}(f, x)-\frac{2}{n(n+1)} \sum_{k=0}^{n-1}(k+1) \sigma_{k}(f, x) .
$$

Hence, (2.4) implies (2.8).

Remark 2.2. For $\frac{4}{3}<p<4$ Pollard's result and (2.7) already imply (2.8).

\section{The Equivalence Result}

We can now state and prove our equivalence result.

Theorem 3.1. For $f \in L_{p}[-1,1], R_{n}(f, x)$ given by (2.6) and the $K$-functional

$$
K\left(f, t^{2}\right)_{p} \equiv K_{2}\left(f, t^{2}\right)_{p}=\inf _{g \in C^{2}}\left(\|f-g\|_{p}+t^{2}\|P(D) g\|_{p}\right),
$$

we have

$$
\left\|R_{n} f-f\right\|_{p} \sim K\left(f, n^{-2}\right)_{p}
$$

where $A_{n} \sim B_{n}$ if there exists $C$ such that $C^{-1} A_{n}<B_{n}<C A_{n}$.

Proof. As polynomials are dense in $L_{p}$ or in $C[-1,1]$ when $p=\infty,(2.8)$ implies

$$
\lim _{n \rightarrow \infty}\left\|R_{n} f-f\right\|_{p}=0 .
$$

This follows from $\lim _{n \rightarrow \infty} R_{n} P_{k}=\lim _{n \rightarrow \infty}\left(1-\frac{k(k+1)}{n(n+1)}\right) P_{k}=P_{k}$ and

$$
\left\|R_{n} f-f\right\|_{p} \leq\left\|R_{n}(f-P)\right\|_{p}+\|f-P\|_{p}+\left\|R_{n} P-P\right\|_{p} .
$$

To prove the direct result we choose $g \in C^{2}[-1,1]$ such that

$$
\|f-g\|_{p}+n^{-2}\|P(D) g\|_{p} \leq 2 K\left(f, n^{-2}\right)_{p}
$$

and write

$$
\begin{aligned}
\left\|R_{n} f-f\right\|_{p} & \leq\left\|R_{n}(f-g)-(f-g)\right\|_{p}+\left\|R_{n} g-g\right\|_{p} \\
& \leq(C+1) 2 K\left(f, n^{-2}\right)_{p}+\left\|R_{n} g-g\right\|_{p} .
\end{aligned}
$$


To estimate the second term we write

$$
\begin{aligned}
\left\|R_{n} g-g\right\| & \leq\left\|R_{n}^{2} g-R_{n} g\right\|+\left\|R_{n}^{2} g-g\right\| \\
& \leq\left\|R_{n}^{2} g-R_{n} g\right\|+\sum_{m=n}^{\infty}\left\|R_{m}^{2} g-R_{m+1}^{2} g\right\|,
\end{aligned}
$$

as (2.8) and (3.3) imply also $\lim _{n \rightarrow \infty}\left\|R_{n}^{2} g-g\right\|=0$ for any $g \in C^{2}[-1,1]$. We now observe that for any $f \in L_{1}[-1,1]$ (so that the $a_{k}$ 's are defined)

$$
n(n+1) R_{n}\left(R_{n} f-f\right)=P(D) R_{n} f,
$$

and that

$$
P(D) R_{n} g=R_{n} P(D) g \quad \text { for } g \in C^{2}[-1,1] .
$$

Therefore,

$$
\left\|R_{n}^{2} g-R_{n} g\right\|_{p} \leq \frac{C}{n(n+1)}\|P(D) g\|_{p} .
$$

As $R_{\ell} R_{m} f=R_{m} R_{\ell} f$, we have

$$
\left\|R_{m}^{2} g-R_{m+1}^{2} g\right\|_{p} \leq\left\|R_{m}^{2} g-R_{m+1} R_{m} g\right\|_{p}+\left\|R_{m+1}^{2} g-R_{m} R_{m+1} g\right\|_{p} .
$$

We now note that for $f \in L_{1}[-1,1]$

$$
\begin{aligned}
R_{m}^{2} f-R_{m+1} R_{m} f & =-\frac{2}{m(m+1)(m+2)} P(D) R_{m} f, \\
R_{m+1}^{2} f-R_{m} R_{m+1} f & =\frac{2}{m(m+1)(m+2)} P(D) R_{m+1} f .
\end{aligned}
$$

Recalling (3.5), we have

$$
\begin{aligned}
\sum_{m=n}^{\infty}\left\|R_{m}^{2} g-R_{m+1}^{2} g\right\|_{p} & \leq 4 C\|P(D) g\|_{p} \sum_{m=n}^{\infty} \frac{1}{m^{3}} \\
& \leq \frac{2 C}{n^{2}}\|P(D) g\|_{p},
\end{aligned}
$$

which, together with the above, yields

$$
\left\|R_{n} f-f\right\|_{p} \leq(C+1) 2 K\left(f, n^{-2}\right)_{p}+3 C \cdot 2 K\left(f, n^{-2}\right)_{p},
$$

and this is the direct estimate of our equivalence.

To prove the converse result, which is a strong converse inequality of type A in the terminology of [7], we note that using (3.1),

$$
K\left(f, n^{-2}\right)_{p} \leq\left\|f-R_{n} f\right\|_{p}+\frac{1}{n^{2}}\left\|P(D) R_{n} f\right\|_{p} .
$$

Using (3.4), we now have

$$
\left\|P(D) R_{n} f\right\| \leq n(n+1) C\left\|R_{n} f-f\right\|_{p},
$$

which completes the proof. 
Corollary 3.2. For $f \in L_{p}[-1,1], 1 \leq p \leq \infty$, we have

$$
\left\|\left(R_{n}-I\right)^{r} f\right\|_{p} \sim \inf _{g \in C^{2 r}[-1,1]}\left(\|f-g\|_{p}+n^{-2 r}\left\|P(D)^{r} g\right\|_{p}\right) \equiv K_{2 r}\left(f, n^{-2 r}\right)_{p} .
$$

Proof. We use Theorems 10.2 and 10.3 of [7] (the latter with some obvious modifications) for the direct result and Theorem 10.4 of [7] for the converse result. That is, the necessary inequalities needed for this extension were already proved earlier in this section.

\section{Further RESUlts AND Generalizations}

We first deduce as a corollary from Theorem 3.1 and Corollary 3.2 the following "realization" result.

Theorem 4.1. For $f \in L_{p}[-1,1], 1 \leq p \leq \infty, R_{n}$ given by (2.6),

$$
K_{2 r}\left(f, t^{r}\right)_{p} \equiv \inf _{g \in C^{2 r}[-1,1]}\left(\|f-g\|_{p}+t^{r}\left\|P(D)^{r} g\right\|_{p}\right)
$$

and

$$
L_{n, r} f=\sum_{k=1}^{r}(-1)^{k+1}\left(\begin{array}{l}
r \\
k
\end{array}\right) R_{n}^{k} f
$$

we have

$$
\left\|f-L_{n, r} f\right\|_{p}+n^{-2 r}\left\|P(D)^{r} L_{n, r} f\right\|_{p} \sim K_{2 r}\left(f, n^{-2 r}\right)_{p} .
$$

Proof. Using Corollary 3.2, all we have to show is

$$
n^{-2 r}\left\|P(D)^{r} L_{n, r} f\right\|_{p} \leq C K_{2 r}\left(f, n^{-2 r}\right)_{p} .
$$

The identities (3.4) and (3.5) used repeatedly imply

$$
P(D)^{r} R_{n}^{k} f=(n(n+1))^{r} R_{n}^{k}\left(R_{n}-I\right)^{r} f .
$$

Hence,

$$
\begin{aligned}
n^{-2 r}\left\|P(D)^{r} R_{n}^{k} f\right\|_{p} & \leq C_{1}\left\|\left(R_{n}-I\right)^{r} f\right\|_{p} \\
& \leq C_{2} K_{2 r}\left(f, n^{-2 r}\right)_{p},
\end{aligned}
$$

from which (4.4) follows easily.

The realization implies the natural relation for hierarchy of measures of smoothness on the $K$-functionals. Hence, realization, though weaker in a sense than (2.8), is important. The first result actually follows already from Corollary 3.2. 
Corollary 4.2. For $K_{2 r}\left(f, n^{-2 r}\right)_{p}$ given in (4.1)

$$
K_{2 r+2}\left(f, n^{-2(r+1)}\right)_{p} \leq C K_{2 r}\left(f, n^{-2 r}\right)_{p} .
$$

Proof. In view of Corollary 3.2 we just have to observe that

$$
\begin{aligned}
\left\|f-L_{n, r+1} f\right\|_{p} & \leq\left\|\left(I-R_{n}\right)\left(f-L_{n, r} f\right)\right\|_{p} \\
& \leq\left\|f-L_{n, r} f\right\|_{p}+\left\|R_{n}\left(f-L_{n, r} f\right)\right\|_{p},
\end{aligned}
$$

which, using (2.8), implies (4.5).

The Marchaud-type inequality also follows.

Theorem 4.3. For $K_{2 r}(f, t)_{p}$ given in (4.1) we have

$$
K_{2 r}\left(f, t^{2 r}\right)_{p} \leq C t^{2 r}\left(\int_{t}^{1} \frac{K_{2 r+2}\left(f, u^{2(r+1)}\right)_{p}}{u^{2 r+1}} d u\right) .
$$

Proof. We write for $n=[1 / t]$

$$
\begin{aligned}
K_{2 r}\left(f, t^{2 r}\right)_{p} & \leq\left\|f-L_{n, r+1} f\right\|_{p}+t^{2 r}\left\|P(D)^{r} L_{n, r+1} f\right\|_{p} \\
& \leq C_{1} K_{2 r+2}\left(f, n^{-2 r-2}\right)_{p}+t^{2 r}\left\|P(D)^{r} L_{n, r+1} f\right\|_{p} .
\end{aligned}
$$

We now choose $k$ so that $2^{k} \leq n<2^{k+1}$ and write

$$
L_{n, r+1} f=L_{n, r+1} f-L_{2^{k}, r+1} f+\sum_{\ell=1}^{k}\left(L_{2^{\ell}, r+1} f-L_{2^{\ell-1}, r+1} f\right)+L_{1, r+1} f .
$$

Using $L_{1, r+1} f=C$, and hence $P(D)^{r} L_{1, r+1} f=0$, and

$$
\left\|L_{2^{\ell}, r+1} f-L_{2^{\ell-1}, r+1} f\right\|_{p} \leq 2 C_{1} K_{2 r+2}\left(f, 2^{-2(r+1)(\ell-1)}\right)_{p},
$$

we have, using the Markov-Bernstein inequality,

$$
\begin{aligned}
K_{2 r}\left(f, t^{2 r}\right)_{p} \leq & C_{1} K_{2 r+2}\left(f, n^{-2 r-2}\right)_{p}+t^{2 r} \sum_{\ell=0}^{k-1} C_{2} 2^{2 \ell r} 2 C_{1} K_{2 r+2}\left(f, 2^{-2(r+1) \ell}\right)_{p} \\
& +2 C_{2} C_{1} t^{2 r} n^{2 r} K_{2 r+2}\left(f, 2^{-2(r+1) k}\right)_{p} .
\end{aligned}
$$

Using monotonicity of $K_{2 r+2}(f, u)_{p}$ and of $u^{\alpha}$, we now complete the proof.

We note that we do not have a free term as usual in Marchaud-type inequalities. While this follows from the proof above, it is related intuitively to the fact that if for some $r, P(D)^{r} g=0$ and $g \in C^{2 r}(-1,1)$, then $g(x)=A$.

The near best polynomial approximant $P_{n}$ which is a polynomial satisfying

$$
\left\|f-P_{n}\right\|_{p} \leq C E_{n}(f)_{p},
$$

where $E_{n}(f)_{p}=\inf _{P \in \Pi_{n}}\|f-P\|_{p}$, can also serve as a realization of the $K$-functional $K_{2 r}\left(f, t^{2 r}\right)_{p} \|_{p}$. 
Theorem 4.4. For $1 \leq p \leq \infty$ and $P_{n}$ given by (4.7) for the given $p$ we have

$$
\left\|f-P_{n}\right\|_{p}+n^{-2 r}\left\|P(D)^{r} P_{n}\right\|_{p} \sim K_{2 r}\left(f, n^{-2 r}\right)_{p} .
$$

Proof. Obviously,

$$
\left\|f-P_{n}\right\|_{p} \leq C\left\|f-L_{n, r} f\right\|_{p} \leq C K_{2 r}\left(f, n^{-2 r}\right)_{p} .
$$

Furthermore,

$$
n^{-2 r}\left\|P(D)^{r} P_{n}\right\|_{p} \leq n^{-2 r}\left\|P(D)^{r} L_{n, r} f\right\|_{p}+n^{-2 r}\left\|P(D)^{r}\left(P_{n}-L_{n, r} f\right)\right\|_{p} .
$$

Since (4.9) yields the estimate

$$
\left\|P_{n}-L_{n, r} f\right\|_{p} \leq 2 C K_{2 r}\left(f, n^{-2 r}\right)_{p},
$$

we complete the proof using the Markov-Bernstein inequality.

One should note that $P_{n}$ satisfying (4.7) may also serve as a realization of $K_{r, \varphi}\left(f, n^{-r}\right)_{p}[6$, Chapter 7] and that this does not imply equivalence of $K_{2 r}\left(f, n^{-2 r}\right)_{p}$ and $K_{2 r, \varphi}\left(f, n^{-2 r}\right)_{p}$.

We can also define delayed means using $R_{n} f$ by

$$
V_{n} f=2 \frac{2 n+1}{3 n+1} R_{2 n} f-\frac{n+1}{3 n+1} R_{n} f
$$

such that

$$
V_{n} P=P \quad \text { for } \quad P \in \Pi_{n}
$$

Obviously, for $n \geq n_{0}$

$$
\left\|V_{n} f\right\|_{p} \leq 2 C\|f\|_{p}
$$

where $C$ is given by Lemma 2.1 .

This obviously implies (for $n \geq n_{0}$ )

$$
\left\|V_{n} f-f\right\|_{p} \leq(2 C+1) E_{n}(f)_{p},
$$

which makes $V_{n} f$ a near best polynomial approximant.

In Theorem 4.4 we may replace $P_{n}$ by $V_{n} f$ since

$$
\left\|f-V_{n} f\right\|_{p} \leq C_{1} E_{n}(f)_{p}
$$

and since for $P_{n} f$ satisfying (4.4) we have, using the Markov-Bernstein inequality $\left(V_{n} f \in \Pi_{2 n}\right.$ and $\left.P_{n} \in \Pi_{n} \subset \Pi_{2 n}\right)$,

$$
\begin{aligned}
\frac{1}{n^{2 r}}\left\|P(D)^{r}\left(P_{n}-V_{n} f\right)\right\|_{p} & \leq C_{2}\left\|P_{n}-V_{n} f\right\|_{p} \\
& \leq C_{3} E_{n}(f)_{p} \leq C_{4} K_{2 r}\left(f, n^{-2 r}\right)_{p} .
\end{aligned}
$$




\section{RELATION With BEST POLYNOMIAL APPROXIMATION}

One observes that

$$
E_{n}(f)_{p} \leq\left\|\left(R_{n}-I\right)^{r} f\right\|_{p}
$$

Hence, using (3.8),

$$
E_{n}(f)_{p} \leq C K_{2 r}\left(f, n^{-2 r}\right)_{p}
$$

The estimate (5.2) has the following matching weak converse inequality.

Theorem 5.1. The K-functional $K_{2 r}\left(f, t^{2 r}\right)_{p}$ given in (1.2) is estimated by the rate of best polynomial approximation in the weak-type inequality given by

$$
K_{2 r}\left(f, t^{2 r}\right)_{p} \leq C t^{2 r} \sum_{0 \leq n \leq 1 / t}(n+1)^{2 r-1} E_{n}(f)_{p} .
$$

Proof. The proof is quite routine, choosing $\ell$ such that $\ell=\max \left\{k ; 2^{k} \leq 1 / t\right\}$ and $g$ to be $P_{2^{k}}$, the best $2^{k}$ degree polynomial approximation to $f$ in $L_{p}[-1,1]$. We then expand $P_{2^{\ell}}$ by

$$
P_{2^{\ell}}(x)=\sum_{k=0}^{\ell}\left(P_{2^{k+1}}(x)-P_{2^{k}}(x)\right)+P_{1}(x) .
$$

The Markov-Bernstein inequality implies

$$
\left\|P(D)^{r} P_{m}\right\|_{p} \leq C m^{2 r}\left\|P_{m}\right\|_{p} \quad \text { for } P_{m} \in \Pi_{m} .
$$

This yields

$$
K_{2 r}\left(f, t^{2 r}\right)_{p} \leq C t^{2 r}\left(\sum_{1 \leq 2^{k} \leq \frac{1}{t}}\left(2^{k}\right)^{2 r} E_{2^{k}}(f)_{p}+E_{0}(f)_{p}\right),
$$

which implies (5.3), using the monotonicity of $E_{n}(f)_{p}, K_{2 r}\left(f, t^{2 r}\right)_{p}$ and $m^{2 r}$.

\section{REFERENCES}

1. R. Askey, Orthogonal polynomials and special functions, Regional Conference Series in Applied Mathematics, vol. 21, Society for Industrial and Applied Mathematics, Philadelphia, PA, 1975. MR 58:1288

2. R. Askey and I.I. Hirschman, Mean summability for ultraspherical polynomials, Math. Scand. 12 (1963), 167-177. MR 29:1497

3. W. Chen and Z. Ditzian, Strong converse inequality for Kantorovich polynomials, Const. Approx. 10 (1994), 95-106. MR 94k:41039

4. W. Chen, Z. Ditzian, and K. Ivanov, Strong converse inequality for the Bernstein-Durrmeyer operator, J. Approx. Theory 75 (1993), 25-43. MR 94h:41047

5. M. Derrienic, Sur l'approximation de fonctions intégrales sur $[0,1]$ par des polynômes de Bernstein modifies, J. Approx. Theory 31 (1981), 325-343.

6. Z. Ditzian and V. Totik, Moduli of smoothness, Springer-Verlag, 1987. MR 89h:41002

7. Z. Ditzian and K. Ivanov, Strong converse inequalities, J. d'Analyse Math. 61 (1993), 61-111. MR 94m: 41038

8. H. Pollard, The mean convergence of orthogonal series I, Trans. Amer. Math. Soc. 62 (1947), 387-403. MR 9:280d

9. V. Totik, Approximation by Bernstein polynomials, Amer. J. Math. 116 (1994), 995-1018. CMP 94:16

Department of Mathematics, University of Alberta, Edmonton, Alberta, Canada T6G 2G1 